(c) 2016 IEEE. Personal use of this material is permitted. Permission from IEEE must be obtained for all other uses, in any current or future media, including reprinting/republishing this material for advertising or promotional purposes, creating new collective works, for resale or redistribution to servers or lists, or reuse of any copyrighted component of this work in other works.

Digital Object Identifier (DOI): 10.1109/EPE.2016.7695606

European Conference on Power Electronics and Applications (EPE'16 ECCE Europe)

Wide frequency range medium-voltage grid impedance analysis by current injection of a multi-MW power converter

Frederik Hahn

Sebastian Brüske

Berthold Benkendorff

Giampaolo Buticchi

Friedrich W. Fuchs

Marco Liserre

Suggested Citation

F. Hahn, S. Brueske, B. Benkendorff, G. Buticchi, F. W. Fuchs, and M. Liserre, "Wide frequency range medium-voltage grid impedance analysis by current injection of a multi-MW power converter," in $18^{\text {th }}$ European Conference on Power Electronics and Applications (EPE'16 ECCE Europe), Sept 2016, pp. 1-10. 


\title{
Wide Frequency Range Medium-Voltage Grid Impedance Analysis by Current Injection of a Multi-MW Power Converter
}

\author{
Frederik Hahn, Sebastian Brüske, Berthold Benkendorff, \\ Giampaolo Buticchi, Friedrich W. Fuchs, Marco Liserre \\ Chair of Power Electronics \\ University of Kiel \\ Kaiserstraße 2 \\ Kiel, Germany \\ Phone: +49 (0) 431/880-6105 \\ Fax: +49 (0) 431/880-6103 \\ Email: $\{$ frha,seb,bb,gibu,fwf,ml $\} @$ tf.uni-kiel.de \\ URL: http://www.pe.tf.uni-kiel.de/en
}

\section{Acknowledgments}

The authors would like to thank the Federal Ministry for Economic Affairs and Energy of Germany for Funding this project.

\section{Keywords}

$\ll$ Impedance measurement $\gg, ~ «$ High frequency power converter $\gg, ~ «<$ Power quality $\gg$

\begin{abstract}
More and more energy is fed especially by decentralized converters from renewable energy sources into the grid. Therefore, it is important to know the grid behavior at a wide frequency range from the fundamental frequency up to the kHz-range. This paper presents an advanced impedance mediumvoltage measurement system with a high frequency range for an electrical medium-voltage grid. A sinusoidal current of a predefined frequency is injected into the grid. With this, the grid impedance is calculated from the measured magnitude and phase of the injected current and the resulting voltage. The measurements will be executed at different frequencies to produce approximate continuous impedance versus frequency characteristic of the grid at the measured coupling point. Results of a ready to use, lowvoltage impedance measurement system are discussed, and the design considerations the basic concept and first simulation results of the developed medium-voltage system are presented in this paper.
\end{abstract}

\section{Introduction}

The electrical grid is changing considerably due to the steadily growing integration of decentralized energy resources (DERs), such as power generation from wind and solar energy. Besides the feed-in of active and reactive power, the DERs introduce additional filter elements, such as inductors and capacitors, that are influencing the grid impedance. Due to the switching behavior of power electronics DERs can be seen as harmonic current sources, which are interacting with the grid impedance inducing harmonic content into the grid voltage. Consequently, the knowledge of the grid impedance is of high importance for the generation and limitation of harmonics, for setting voltage harmonic limitations in grid standards, and for the design of grid connected power converters. Moreover, the control of the power converters of the DERs has to be adapted to the grid impedance to guarantee stability and sufficient dynamic behavior. 


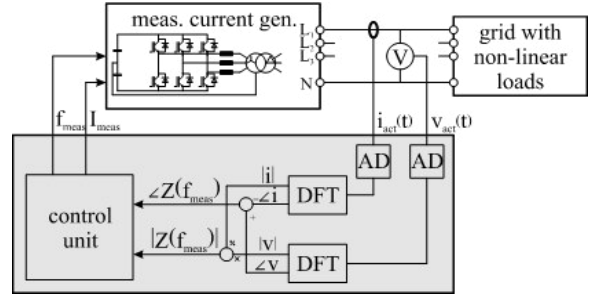

(a)

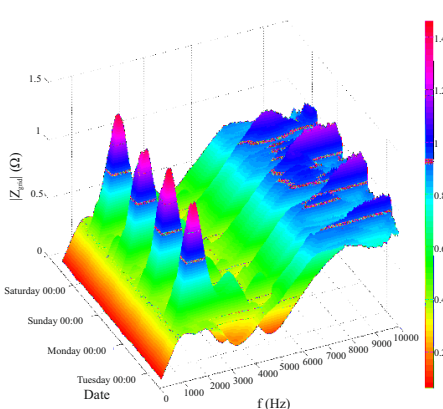

(b)

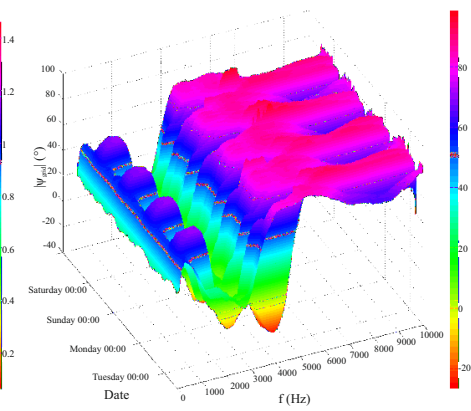

(c)

Figure 1: (a) Setup for the LV grid impedance measurement, $(b, c)$ : Grid impedance measurement in a public LV grid versus time and frequency for (b) magnitude and (c) for phase angle

In the conventional approach for the grid connection of DERs, the grid impedance at the point of connection (PoC) is calculated at the fundamental grid frequency based on shortcircuit power [1]. In special cases grid simulation software is used to obtain more precise results. Nevertheless, this approach is insufficient since the grid impedance between $0.1 \mathrm{kHz}$ and $10 \mathrm{kHz}$, the range of the dominant harmonic frequencies of the DERs, has to be known. This is valid for both low-voltage (LV) grids with smaller DERs and medium-voltage (MV) grids, where wind and solar parks are connected. For LV grids, measurements of the grid impedance in the interesting frequency range of $0.1 \mathrm{kHz}$ to $10 \mathrm{kHz}$ under varying grid conditions are published [2, 3, 4]. In most publications, the focus is on the development of the analysis system, while measurement results are not addressed in greater depth. For MV grids only very few measurements are published, for example [5,6]. A deeper knowledge in this field is required for appropriate and optimized operation of the electrical grid.

In this paper, a concept for a system and a method to analyze the MV grid in regard to grid integration of DERs is presented. A project has been started to develop and set up an MV grid analysis system and to perform extensive grid measurements. A single step-by-step frequency current feed-in method has been chosen, which offers accurate results over a wide frequency range. A concept of the overall system to analyze the grid impedance in MV grids is presented with the focus on the converter design of the measuring current generator, where a power of $2 \mathrm{MVA}$ to $3 \mathrm{MVA}$ is required. Additional features of the converter will be reactive power supply and active power filtering (APF). Possible realizations by twolevel inverters and by NPC inverters are described and compared to optimize the switching frequency and the harmonic content. The results can be applied for other grid connected converters like static synchronous generator (STATCOM) or APF.

This paper is organized as follows: The first section gives references to successful operated grid measurements in the LV grid and describes the transfer of measurement methods to the MV case. The measurement concept for MV grid impedance is presented in the next section. The third section contains the converter design for the measurement current generator. In the following section simulation results of the generator system are shown. The final section gives the conclusion.

\section{Low-Voltage Grid Impedance Measurement}

The impedance measurement of energized power grids at non-fundamental frequencies is a well-known approach. Generally, the methods presented in the literature only differ in the measurement frequency band and its frequency resolution. Beyond that, impedance measurement methods can be divided into passive and active methods. A simple passive approach to derive the impedance at the harmonic and fundamental frequencies is the steady-state measurement of the line voltage and phase current before and after the grid connection of a known load [7]. Another passive method is based on the excitation of the LCL-filter resonance in steady-state operations to estimate the grid impedance [8]. In active methods, non 
fundamental frequency signals are injected into a power system, which are either pulsed signals (polyfrequency) [1,9] or single frequency signals generated with special filter equipment (mono-frequency) [10]. In comparison to active methods, passive methods have the drawback that the grid impedance can only be measured at frequencies present in the grid voltage. If the grid voltage amplitude is not large enough at the specific harmonic frequency to adhere to the measurement accuracy, it can be amplified by the harmonic current injection caused by the non-linearity of the known load [11]. In both cases the measurement frequencies are mainly odd harmonics. In active, poly-frequency measurement systems the power is split among the full measurement frequency band leading to a decreased measurement power at the single frequency, which results in a lower signal-to-noise ratio in comparison to methods based on mono-frequency measurements [12]. Further advantages of mono-frequency measurement systems are the capability to arbitrarily select the measurement frequencies and the number of samples as well as to set the maximum measurement current at each frequency in dependence of the grid conditions. However, mono-frequency measurements have to be repeated for each single frequency resulting in a higher measurement time that can lead to falsified results if the grid conditions change within the measurement period.

A successful LV impedance measurement was carried out with a three-phase $15 \mathrm{kVA}$ grid connected VSC, which is shown in Figure 1a. With this converter an active, mono-frequency measurement with a range from $100 \mathrm{~Hz}$ up to $10 \mathrm{kHz}$ [12] is realized to measure the LV grid impedance with high accuracy over a wide frequency range. The measurement results are used for optimizing the grid integration of DERs, and for grid modeling, which gives information to DER manufacturers and operators as well as energy suppliers about the grid behavior contributing to the design of products as well as to standardization requirements for DERs. Measurements were taken in a public LV power system in northern Germany [13]. Figures $1 b, c$ indicate that within the four days of measurements the frequency depending impedance of the considered $\mathrm{PoC}$ is not constant but varies in time with a conspicuous day profile. Several resonances can be found in the magnitude of $Z_{\mathrm{PoC}}$ in Figure $1 \mathrm{~b}$. The main resonance occurs at $1800 \mathrm{~Hz}$ with a magnitude of $Z_{\mathrm{PoC}}=1.3 \Omega$ around noon. The phase angle of $Z_{\mathrm{PoC}}$ is illustrated in Figure $1 \mathrm{c}$ showing that the phase angle at $50 \mathrm{~Hz}$ is predominantly resistive at an angle of about $20^{\circ}$. During nighttime the phase angle decreases in a frequency range from $1.6 \mathrm{kHz}$ and $5 \mathrm{kHz}$ at a minimum angle of $-25^{\circ}$. From these results it can be concluded that the grid impedance is highly variable, which makes it necessary to measure the grid impedance also in the MV grid.

\section{Medium-Voltage Grid Impedance Measurement System}

An active, mono-frequency measurement system is chosen to provide high measurement accuracy over a wide frequency range even in strong grid conditions. The hardware concept is described in this section. The main challenge is the high switching frequency up to $50 \mathrm{kHz}$ at high power levels. Therefore, inverters with LV ratings are chosen, as they can be designed for this switching frequency range. For reasons of operability the power level of the single LV converters is chosen to be 200-600 kVA leading to a total number of 6-15 converters connected in parallel in order to reach the required total power of $2 \mathrm{MVA}$ to $3 \mathrm{MVA}$. Special challenges for parallel inverters are the synchronization and control of inverters and the suppression of circulating currents.

\section{Power Converter Architecture}

The overall concept is depicted in Figure 2a. The current generator consists of parallel converters providing a measurement current with a frequency between $100 \mathrm{~Hz}$ and $10 \mathrm{kHz}$. The generator is connected to the MV grid $(20 \mathrm{kV})$ by a multi MVA transformer in star-star configuration (short circuit voltage $u_{\mathrm{k}}=2.5 \%$ ) to minimize the voltage level which has to be generated by the inverters. A grid excitation for measurement of $1 \%$ of the MV is necessary. The current generator is fed by the LV grid $(50 \mathrm{~Hz})$ whereby a LV transformer is used to reduce the voltage level at the LV side. The voltages and currents are measured and analyzed by a Fourier analysis to determine the grid impedance. 
Table I: Basic data for $20 \mathrm{kV}$ MV grid for the connection of decentralized energy systems [14]

\begin{tabular}{ccccccc}
\hline Case & $V_{\mathrm{MV}, \Delta}[\mathrm{kV}]$ & $S_{\mathrm{k}}[\mathrm{MVA}]$ & $\Psi\left[^{\circ}\right]$ & $Z_{\mathrm{k}, \mathrm{MV}}[\Omega]$ & $L_{\mathrm{k}, \mathrm{MV}}[\mathrm{mH}]$ & $R_{\mathrm{k}, \mathrm{MV}}[\Omega]$ \\
\hline Range & $20 \ldots 22$ & $30 \ldots 500$ & $50 \ldots 85$ & & & \\
A & 20 & 30 & 85 & 13.3333 & 42.3 & 1.1621 \\
B & 20 & 500 & 50 & 0.8 & 1.95 & 0.5142 \\
\hline
\end{tabular}

\section{Control and Synchronization}

In order to guarantee an accurate impedance measurement and a safe operation at the same time the $N$ parallel inverters have to be synchronized by a supervisory control system and monitored locally. The control concept is shown in Figure 2b. At inverter level, the current and DC link voltage are measured and being processed by a local field programmable gate array (FPGA). The measured signals are transmitted to the supervisory control unit via fiber optic cables using serial communication. The current controllers are implemented on the fast FPGA of the supervisory control unit. In addition to the current at the measurement frequency, the fundamental frequency is used to control the DC link voltages. Due to the lower dynamics the voltage controllers can be implemented on a microprocessor, of which the outputs are summed up with the references of the non fundamental frequency currents. For grid synchronization, a phase-locked loop is used to track the voltage $v_{\text {pcc }}$, which can be implemented on the microprocessor, likewise. The switching signals are calculated on the FPGA of the supervisory control unit and are transmitted to the local FPGA via fiber optic cables and serial communication, where they are transmitted to the single power converters. In the same way, the switching synchronization of the single inverters can be facilitated.

\section{Converter Design}

The current generator is designed to inject the required measuring current in the MV grid. The minimum and maximum characteristic data of the MV grid $\left(V_{\mathrm{MV}, \Delta}=20 \mathrm{kV}\right)$ in Germany, Russia and Brazil, relevant for this project, are summarized in Table I [14]. The grid characteristics are described by the short-circuit power $S_{\mathrm{k}}$ and the grid impedance angle $\Psi$. The grid impedances $Z_{\mathrm{k}, \mathrm{MV}}$ at $f_{0}=50 \mathrm{~Hz}$ are calculated by equations (1)-(3).

$$
Z_{\mathrm{k}, \mathrm{MV}}^{50 \mathrm{~Hz}}=\frac{V_{\mathrm{MV}, \Delta}^{2}}{S_{\mathrm{k}}} \quad \text { (1) } \quad R_{\mathrm{k}, \mathrm{MV}}^{50 \mathrm{~Hz}}=Z_{\mathrm{k}, \mathrm{MV}} \cdot \cos \Psi \quad \text { (2) } \quad L_{\mathrm{k}, \mathrm{MV}}^{50 \mathrm{~Hz}}=\frac{Z_{\mathrm{k}, \mathrm{MV}} \cdot \sin \Psi}{2 \pi f_{0}}
$$

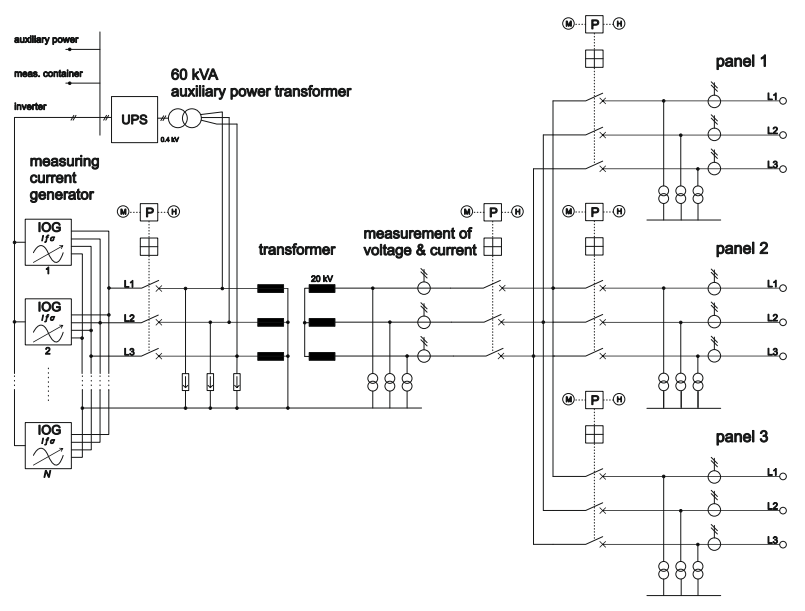

(a)

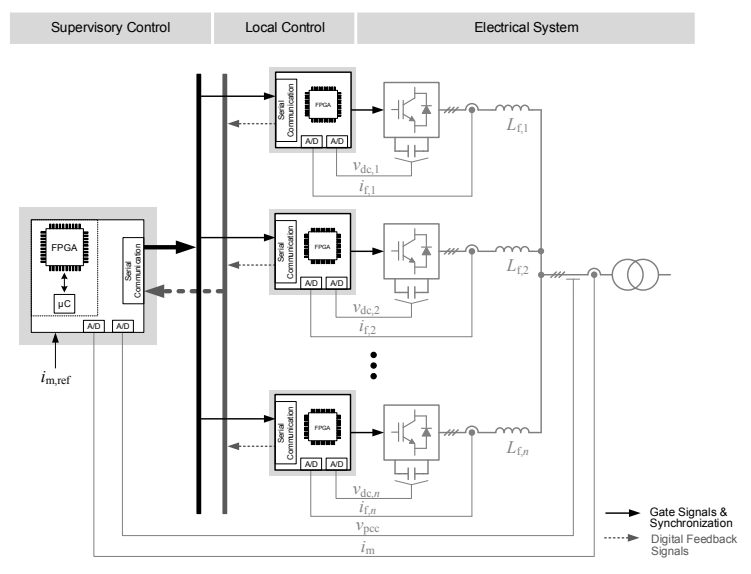

(b)

Figure 2: Overview of MV grid analyzer: (a) block diagram, (b) concept of control and synchronization 


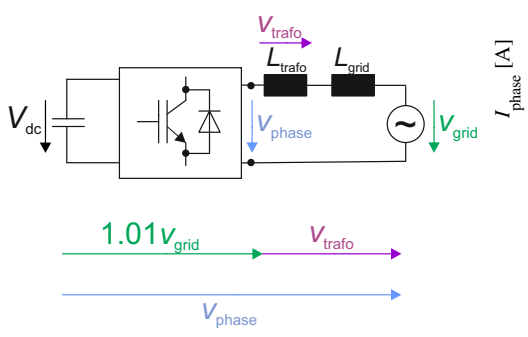

(a)

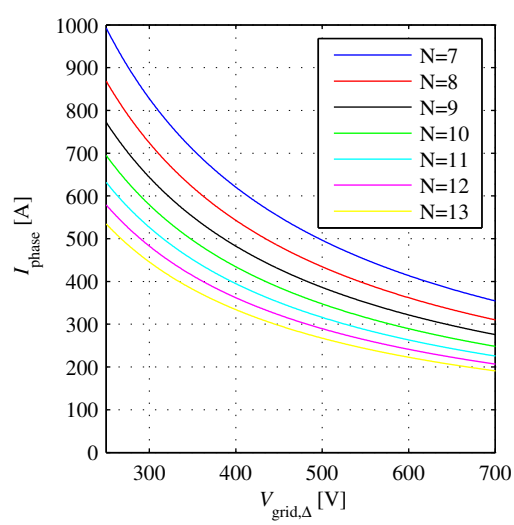

(b)

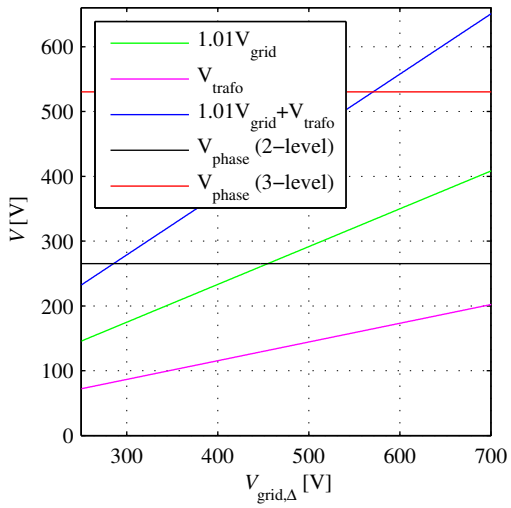

(c)

Figure 3: Schematic diagram of MV grid analyzer: (a) Single phase converter diagram and voltage vector diagram of phase voltage $V_{\text {phase }}$, voltage across transformer impedance $V_{\text {trafo }}$ and grid voltage (LV side) $V_{\text {grid }}$ for reactive power supply, (b) Number of parallel connected inverters to handle expected measurement current at full rated power, (c) Voltage classification of converter: by using 2-level or 3level NPC inverter to excite grid voltage with $1 \%$ at $1 \mathrm{kHz}$

In Table I, case A defines the maximum grid impedance and the maximum grid inductance. The minimum grid impedance and inductance are obtained for Case B. By the simplification that the grid shows an ohmic-inductive behavior up to $100 \mathrm{~Hz}$, it is obtained: $Z_{\mathrm{k} . \mathrm{MV}}^{100 \mathrm{~Hz}}=1.33 \Omega\left(Z_{\mathrm{k}, \mathrm{MV}}^{50 \mathrm{~Hz}}=0.8 \Omega\right)$. This is the minimal impedance for which the converter will be designed. The required current for a grid excitation $\Delta V_{\text {exc }}$ of $1 \%$ can be calculated by equations (4), (5). The current at LV side depends on the transfer ratio $u$ of the transformer according to equation (6).

$$
I_{\mathrm{MV}}=\frac{\Delta V_{\mathrm{exc}, \curlywedge}}{Z_{\mathrm{k}, \mathrm{MV}}(f)} \quad \text { (4) } \quad \Delta V_{\mathrm{exc}, \curlywedge}=0.01 \frac{V_{\mathrm{MV}, \Delta}}{\sqrt{3}} \quad \text { (5) } \quad I_{\mathrm{LV}, \text { total }}=\frac{I_{\mathrm{MV}}}{u}
$$

The current will be provided by $N$ parallel inverters. The full current of one single inverter for different $\mathrm{LV}$ level is depicted in Figure 3b. A rated rms current of $434 \mathrm{~A}$ would be required for 10 parallel inverters connected to a transformer input voltage $V_{\text {grid, } \Delta}$ of $400 \mathrm{~V}$. The parallel converters are summarized in a single phase converter diagram in Figure $3 \mathrm{a}$. The system is related to the LV side. The transformer inductance is calculated by equations (7), (8) whereas $R_{\mathrm{k}, \text { trafo }} \approx 0$.

$$
Z_{\mathrm{k}, \text { trafo }}=u_{k} \cdot \frac{V_{\text {grid }, \curlywedge}}{I_{\mathrm{LV}, \text { total }}}=u_{k} \cdot \frac{V_{\text {grid }, \Delta}^{2}}{S_{\text {total }}} \quad \text { (7) } \quad L_{\mathrm{k}, \text { trafo }}=\frac{Z_{\mathrm{k}, \text { trafo }}}{2 \pi f_{0}}
$$

For proper analysis and due to possible grid resonances the system should be able to inject the full current up to $1000 \mathrm{~Hz}$. IGBTs with a blocking voltage of $1200 \mathrm{~V}$ are considered for the voltage design since 1700 V IGBTs are typically characterized by higher switching losses. The DC link voltage is assumed as $750 \mathrm{~V}$ (two-level converter) and $1500 \mathrm{~V}$ (three-level converter), respectively. The voltage level for the phase voltage is calculated by equation (9).

$$
V_{\text {phase }, \curlywedge}=\frac{V_{\mathrm{dc}}}{2 \cdot \sqrt{2}}
$$

$$
V_{\text {trafo }}=2 \pi f \cdot L_{\mathrm{k}, \text { trafo }} \cdot I_{\mathrm{LV}, \text { total }}
$$


In Figure $3 \mathrm{c}$ the voltage design is illustrated for the worst case that the voltages are in phase. In this operation point the grid voltage, the grid voltage excitation $\left(0.01 \cdot V_{\text {grid }}\right)$ and the voltage drop across the transformer inductance in equation (10) are summed. The sum of these two contributions is required to inject the full current at $1000 \mathrm{~Hz}$. The two-level inverter achieves the required voltage level by selecting a very low transformer input voltage. On the other hand, three-level converters as the NPC converter would profit from a higher voltage level, an additional voltage level and a higher power density [15].

The design and the commissioning of a converter system is always a challenging task. At the Chair of Power Electronics two three-level NPC inverters for different applications have been successfully developed and tested in different operation points in the laboratory. This experience can be used.

The first system is an NPC electrical drive inverter for an electrical vehicle with a rated power of $20 \mathrm{~kW}$ [16]. The inverter is designed for a maximum DC-Link voltage of $750 \mathrm{~V}$, the semiconductors are discrete IGBTs in TO-247 size. The maximum evaluated efficiency is $97.2 \%$.

The second system is a compact LV high power NPC inverter for wind turbine applications with a rated power of approximate $1 \mathrm{MVA}$ [17]. The inverter is designed for a maximum DC-Link voltage of $1500 \mathrm{~V}$, a maximum AC-phase voltage of $950 \mathrm{~V}$ to stay inside the $\mathrm{LV}$ requirements. The semiconductors are NPC phase-leg modules with a rating of $1200 \mathrm{~V} / 600 \mathrm{~A}$. The maximum evaluated efficiency at approx. $820 \mathrm{~kW}$ is $98.7 \%$ for generator-side operation and $98.8 \%$ for grid-side operation.

\section{Simulation Results}

The described converter design for parallel two-level inverters and for parallel NPC inverters is considered in simulation. The power rating of the inverters is due to costs reduced to $60 \%$ achieving $1 \%$ voltage excitation only up to 300 MVA MV grid short circuit power (not up to 500 MVA). The transformer input voltage is selected by $270 \mathrm{~V}$ and $570 \mathrm{~V}$, respectively. By this way, sufficient voltage reserves are provided for the modulation, for additional voltages caused by measuring current and for grid voltage variations. The NPC converter is modulated by phase disposition modulation [18]. The current control is based on PI controllers and optimized by the technical optimum [19]. The DC link voltage is assumed as constant. The relevant simulation parameters are summarized in Table II.

Table II: Simulation Parameters for two-level and three-level NPC inverter

\begin{tabular}{lccc}
\hline Description & Parameter & Two-level inverter & NPC inverter \\
\hline Number of parallel inverters & $N$ & 13 & 7 \\
Current rating (rms) of one inverter & & $300 \mathrm{~A}$ & $300 \mathrm{~A}$ \\
Power rating of one inverter & & $239 \mathrm{kVA}$ & $478 \mathrm{kVA}$ \\
DC link voltage & $V_{\mathrm{dc}}$ & $750 \mathrm{~V}$ & $1500 \mathrm{~V}$ \\
Transformer input voltage (for winding ratio) & $V_{\mathrm{grid}, \Delta}$ & $270 \mathrm{~V}$ & $570 \mathrm{~V}$ \\
Transformer input voltage (for magnetic design) & & $460 \mathrm{~V}$ & $920 \mathrm{~V}$ \\
Transformer inductance & $L_{\mathrm{k}, \text { trafo }}$ & $3.21 \mu \mathrm{H}$ & $14.32 \mu \mathrm{H}$ \\
\hline
\end{tabular}

The phase voltages, the grid voltages and the overall phase currents for two-level and NPC inverters are depicted in Figures $4 \mathrm{a}$ and $4 \mathrm{~b}$, respectively. The full current is generated for a frequency of $100 \mathrm{~Hz}$. The overall current to inject the power is reduced for the parallel NPC inverters due to the increased transformer input voltage. The weighted harmonic spectrum of the overall current (phase 1) is depicted in Figures 5a and 5b. The fundamental frequency becomes visible at $100 \mathrm{~Hz}$. Additionally, there are significant harmonics around the switching frequency of $15 \mathrm{kHz}$ and its multiples. As mentioned before the grid impedance will be determined by a Fourier analysis of voltages and currents. Accordingly, the quality of measurement is not affected by unwanted harmonics. 

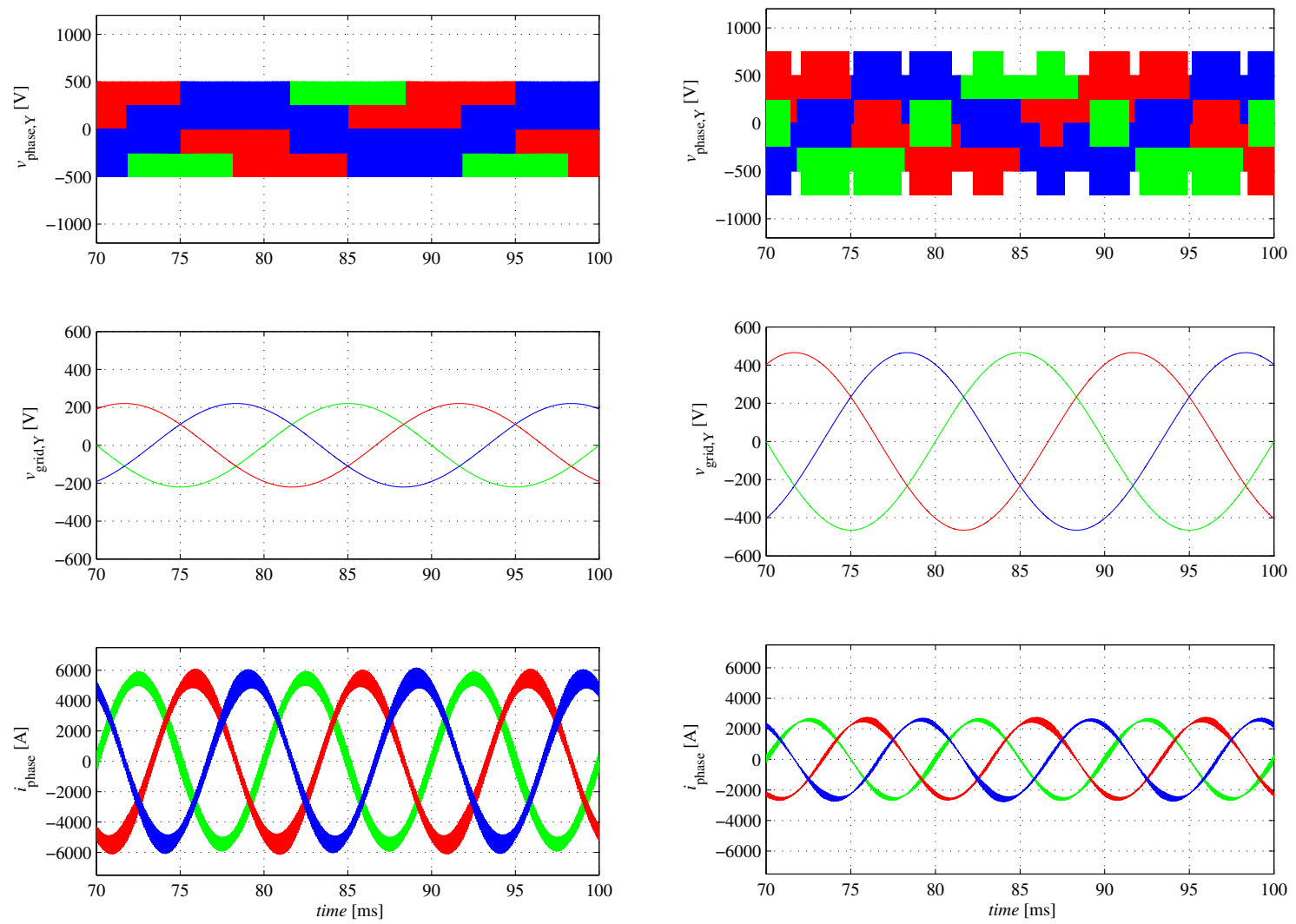

(a)

(b)

Figure 4: Converter phase voltages, grid voltages and overall phase currents (three phases) for full current and a current frequency of $100 \mathrm{~Hz}\left(f_{\mathrm{sw}}=15 \mathrm{kHz}\right)$ : (a) Two-level inverter $\left(\hat{i}_{\text {phase }}=5460 \mathrm{~A}\right)$, (b) NPC inverter $\left(\hat{i}_{\text {phase }}=2587 \mathrm{~A}\right)$

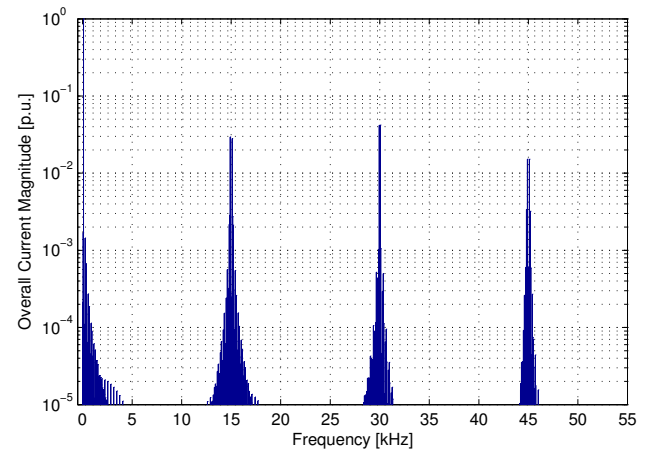

(a)

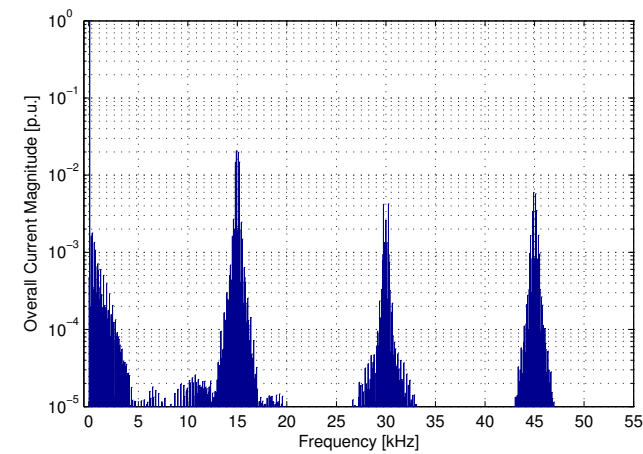

(b)

Figure 5: Weighted harmonic spectrum of overall phase current for a full current and a current frequency of $100 \mathrm{~Hz}\left(f_{\mathrm{sw}}=15 \mathrm{kHz}\right)$ : (a) Two-level inverter $\left(\hat{i}_{\text {phase }}=5460 \mathrm{~A}\right)$, (b) NPC inverter $\left(\hat{i}_{\text {phase }}=2587 \mathrm{~A}\right)$

The full current can be provided up to a current frequency of $1000 \mathrm{~Hz}$. The corresponding phase voltages, grid voltages and overall phase currents are depicted in Figure 6. The generated phase voltages are increased to generate the high voltage drop across the transformer inductance at high frequencies. The harmonic spectra of the overall currents (phase 1) are shown in Figure 7. Again there are significant harmonics around the switching frequency of $15 \mathrm{kHz}$ and its multiples. The increased harmonic content is caused by the reduced ratio between switching and current frequency. 

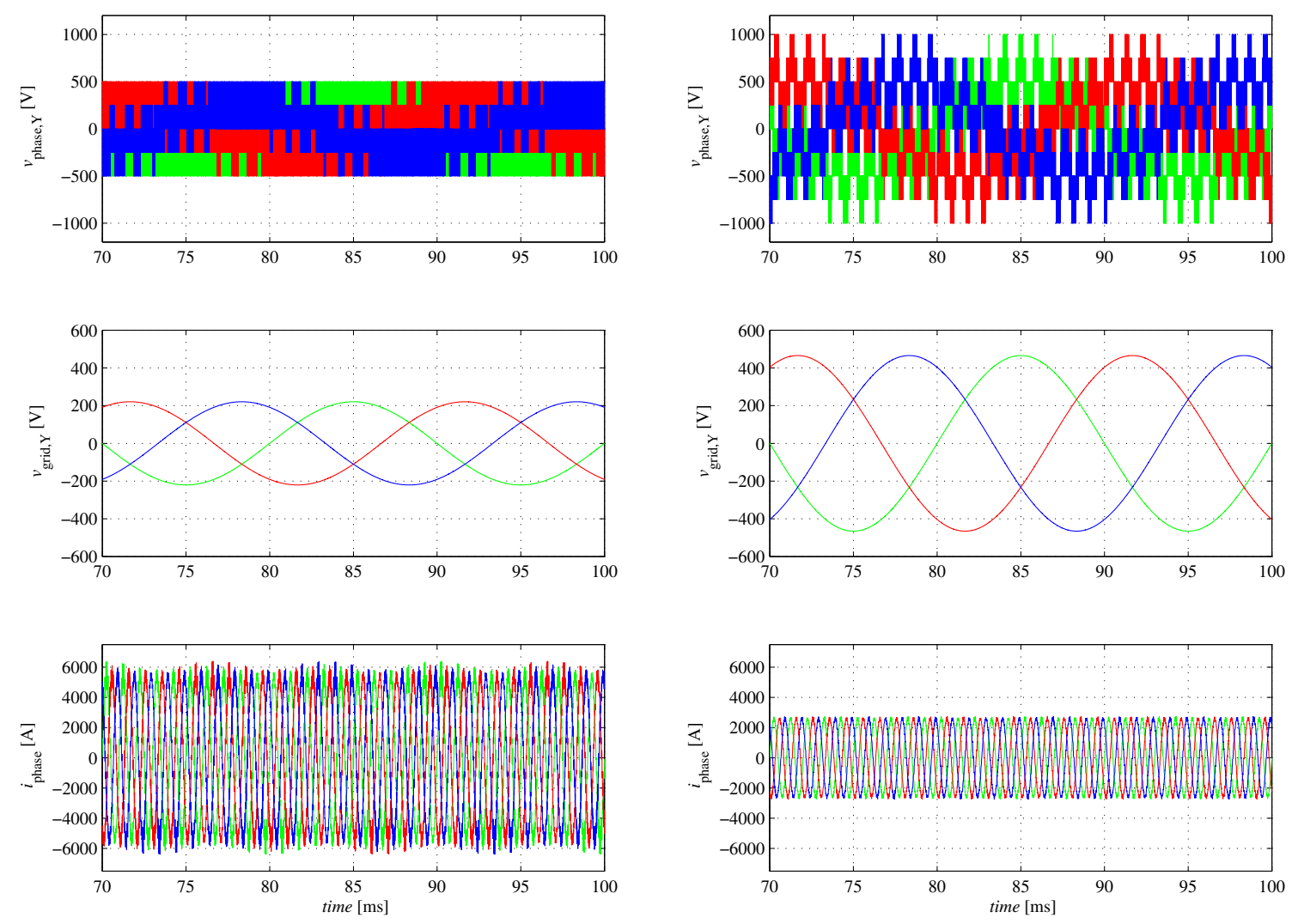

(a)

(b)

Figure 6: Converter phase voltages, grid voltages and overall phase currents (three phases) for full current and a current frequency of $1000 \mathrm{~Hz}\left(f_{\mathrm{sw}}=15 \mathrm{kHz}\right)$ : (a) Two-level inverter $\left(\hat{i}_{\text {phase }}=5460 \mathrm{~A}\right)$, (b) NPC inverter $\left(\hat{i}_{\text {phase }}=2587 \mathrm{~A}\right)$

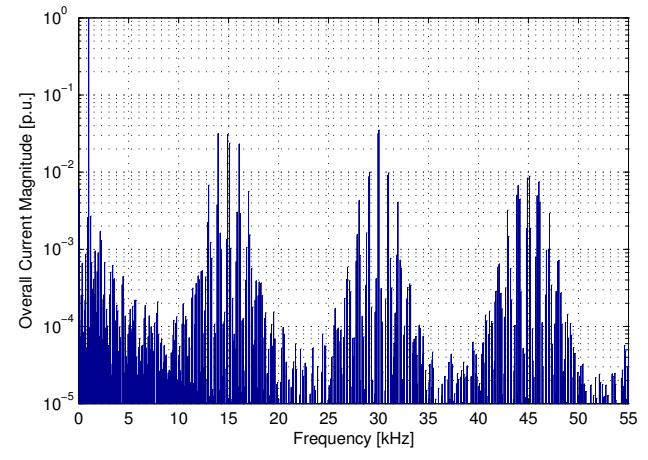

(a)

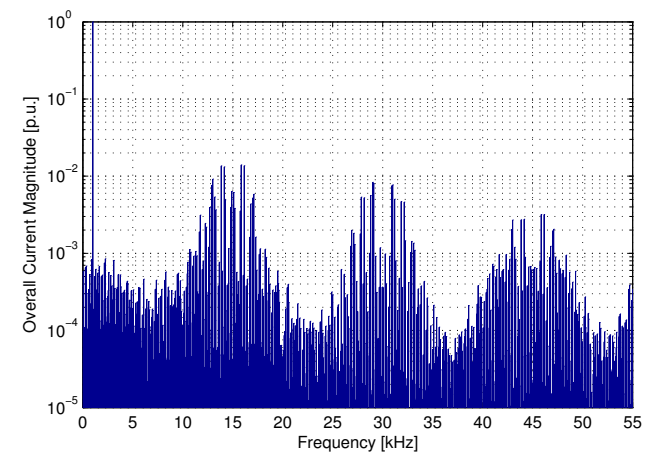

(b)

Figure 7: Weighted harmonic spectrum of overall phase current for a full current and a current frequency of $1000 \mathrm{~Hz}\left(f_{\mathrm{sw}}=15 \mathrm{kHz}\right)$ : (a) Two-level inverter $\left(\hat{i}_{\text {phase }}=5460 \mathrm{~A}\right)$, (b) NPC inverter $\left(\hat{i}_{\text {phase }}=2587 \mathrm{~A}\right)$

Only $10 \%$ of the current has to be provided at the maximum current frequency of $10 \mathrm{kHz}$. For this reason, an increased switching frequency of $30 \mathrm{kHz}$ to $50 \mathrm{kHz}$ becomes feasible. The phase voltages, grid voltages and overall phase currents are depicted in Figure 8. The waveforms are strongly distorted because of the very low ratio between switching and current frequency. However, this is not a critical issue since the system is designed for a much higher rated current. The harmonic spectrum of the overall current is depicted in Figures 9a and 9b. The most significant harmonics are located around the multiples of $10 \mathrm{kHz}$. 

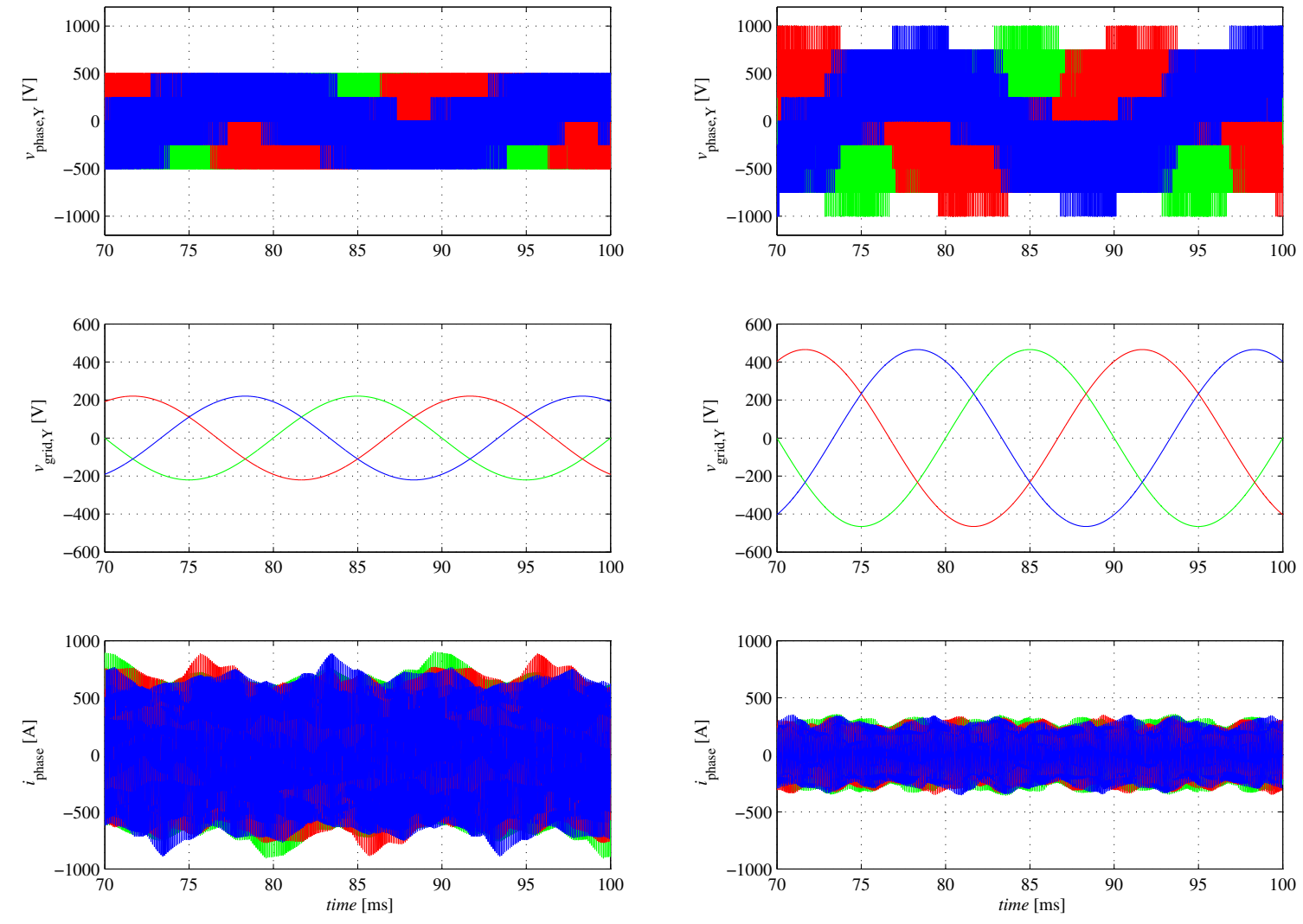

(a)

(b)

Figure 8: Converter phase voltages, grid voltages and overall phase currents (three phases) for full current and a current frequency of $10.000 \mathrm{~Hz}\left(f_{\mathrm{sw}}=50 \mathrm{kHz}\right)$ : (a) Two-level inverter $\left(\hat{i}_{\text {phase }}=546 \mathrm{~A}\right)$, (b) NPC inverter $\left(\hat{i}_{\text {phase }}=259 \mathrm{~A}\right)$

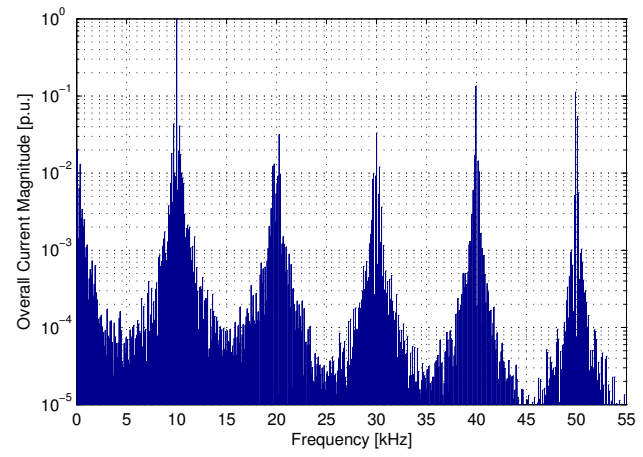

(a)

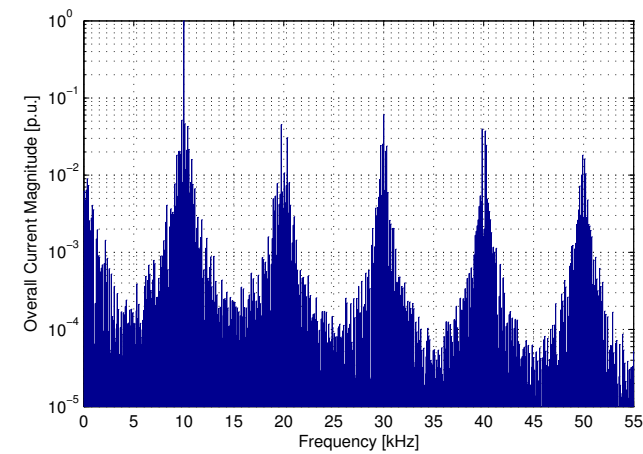

(b)

Figure 9: Weighted harmonic spectrum of overall phase current for full current and a current frequency of $10.000 \mathrm{~Hz}\left(f_{\mathrm{sw}}=50 \mathrm{kHz}\right)$ : (a) Two-level inverter $\left(\hat{i}_{\text {phase }}=546 \mathrm{~A}\right),(\mathrm{b}) \mathrm{NPC}$ inverter $\left(\hat{i}_{\text {phase }}=259 \mathrm{~A}\right)$

\section{Conclusion}

The knowledge of the grid impedance is necessary for a suitable integration of today's and future decentralized energy resources. The grid impedance is time dependent and varies with the frequency, and it is usually not well known. As a consequence, the real grid impedance can only be determined by measurements. A MV grid analysis system based on an MVA power converter is introduced in this paper. The requirement was set up to analyze the MV grid in the important frequency range of $100 \mathrm{~Hz}$ to 
$10 \mathrm{kHz}$. The concept of the measurement system, the basic design considerations and simulations of the measurement current are presented. The designed high power electronic current generator (2 MVA to 3 MVA) with a switching frequency of $50 \mathrm{kHz}$ and the designed measurement system would enable to measure the grid impedance in the wished range with an accuracy higher than $5 \%$.

\section{References}

[1] M. Sumner, B. Palethorpe, and D. Thomas: Impedance measurement for improved power quality- part 1: the measurement technique, IEEE Trans. Power Del., vol. 19, no. 3, pp. 1442-1448, July 2004.

[2] Y.A. Familiant, Jing Huang, K.A. Corzine, and M. Belkhayat: New Techniques for Measuring Impedance Characteristics of Three-Phase AC Power Systems, Power Electronics, IEEE Transactions on, vol. 24, no. 7, pp. 1802- 1810, Jul. 2009.

[3] A. Knop and F.W. Fuchs: High frequency grid impedance analysis by current injection, in Industrial Electronics, 2009. IECON '09. 35th Annual Conference of IEEE, Nov. 2009, pp. 536-541.

[4] S. Guenter, F.W. Fuchs, and H.-J. Hinrichs: A Method to Measure the Network Harmonic Impedance, PCIM Europe 2013, 14-16 May 2013.

[5] J. Xie, Y.X. Feng, and N. Krap: Network Impedance Measurements for Three-phase High-voltage Power Systems, Power and Energy Engineering Conference (APPEEC), 2010 Asia-Pacific, March 2010, pp. 1-5.

[6] M. Jordan, H. Langkowski, Trung Do Thanh, and D. Schulz: Frequency dependent grid-impedance determination with pulse-width-modulation-signals, Compatibility and Power Electronics (CPE), 2011 7th International Conference-Workshop, Jun. 2011, pp. 131-136.

[7] Czarnecki, S. Leszek, and Z. Staroszczyk: On-line measurement of equivalent parameters for harmonic frequencies of a power distribution system and load, IEEE Trans. Instrum. Meas., vol. 45, no. 2, pp. 467472, Apr. 1996.

[8] M. Liserre, F. Blaabjerg, and R. Teodorescu: Grid impedance estimation via excitation of LCL -filter resonance, IEEE Trans. Ind. Appl., vol. 43, no. 5, pp. 1401-1407, Sept 2007.

[9] M. Sumner, B. Palethorpe, and D. Thomas: Impedance measurement for improved power quality- part 2: a new technique for stand-alone active shunt filter control, IEEE Trans. Power Del., vol. 19, no. 3, pp. 14571463, July 2004.

[10] J. Rhode, A. Kelley, and M. Baran: Complete characterization of utilization-voltage power system impedance using wideband measurement, IEEE Trans. Ind. Appl., vol. 33, no. 6, pp. 1472-1479, Nov 1997.

[11] A. de Oliveira, J. C. de Oliveira, J. W. Resende, and M. S. Miskulin: Practical approaches for ac system harmonic impedance measurements, IEEE Transactions on Power Delivery, vol. 6, no. 4, pp. 1721-1726, Oct 1991.

[12] A. Knop, Entwicklung eines schelltaktenden Stromrichters für die Netzimpedanzanalyse im Frequenzbereich bis $10 \mathrm{kHz}$, Dissertation, Universität Kiel, 2013.

[13] L. Jessen, S. Gunter, F. Fuchs, M. Gottschalk, and H.-J. Hinrichs: Measurement results and performance analysis of the grid impedance in different low voltage grids for a wide frequency band to support grid integration of renewables, in Energy Conversion Congress and Exposition (ECCE), 2015 IEEE, Sept 2015, pp. 1960-1967.

[14] B. Valov: Auslegungskonzept des Netzanschlusses von PV-Kraftwerken zwecks Spannungsstabilisierung und voller Nutzung der Netzkapazität, 24. Symposium Photovoltaische Solarenergie, Bad Staffelstein, Mar. 2009.

[15] M. Schweizer and T. Friedli, and J. W. Kolar: Comparative Evaluation of Advanced Three-Phase Three-Level Inverter/Converter Topologies Against Two-Level Systems, IEEE Transactions on Industrial Electronics, vol. 60, no. 12, pp. 5515- 5527, Dec. 2013.

[16] S. Brueske, B. Benkendorff, and F. W. Fuchs: Analysis of the power semiconductor design rating for threelevel neutral-point-clamped inverter based on data sheet parameters, in Power Electronics and Applications (EPE15 ECCE-Europe), 2015 17th European Conference on, Sept 2015, pp. 1- 10.

[17] B. Benkendorff, T. Franke, and F. W. Fuchs: Efficiency verification power circulation method of a high power low voltage npc converter for wind turbines, in PCIM Europe 2016; International Exhibition and Conference for Power Electronics, Intelligent Motion, Renewable Energy and Energy Management; Proceedings of, May 2016, pp. 1-7.

[18] B. Cougo, G. Gateau, T. Meynard, M. Bobrowska-Rafal, and M. Cousineau: PD modulation scheme for three-phase parallel multilevel inverters, IEEE Transactions on Industrial Electronics, vol. 59, no. 2, pp. 690-700, Feb 2012.

[19] V. Blasko and V. Kaura: A new mathematical model and control of a three-phase AC-DC voltage source converter, Jan. 1997, pp. 116-123. 